\title{
Integrating mental health into chronic care in South Africa: the development of a district mental healthcare plan
}

Inge Petersen, Lara Fairall, Arvin Bhana, Tasneem Kathree, One Selohilwe, Carrie Brooke-Sumner, Gill Faris, Erica Breuer, Nomvula Sibanyoni, Crick Lund and Vikram Patel

\section{Background}

In South Africa, the escalating prevalence of chronic illness and its high comorbidity with mental disorders bring to the fore the need for integrating mental health into chronic care at district level.

\section{Aims}

To develop a district mental healthcare plan (MHCP) in South Africa that integrates mental healthcare for depression, alcohol use disorders and schizophrenia into chronic care.

\section{Method}

Mixed methods using a situation analysis, qualitative key informant interviews, theory of change workshops and piloting of the plan in one health facility informed the development of the MHCP.

\section{Results}

Collaborative care packages for the three conditions were developed to enable integration at the organisational, facility and community levels, supported by a human resource mix and implementation tools. Potential barriers to the feasibility of implementation at scale were identified.

\section{Conclusions}

The plan leverages resources and systems availed by the emerging chronic care service delivery platform for the integration of mental health. This strengthens the potential for future scale up.

\section{Declaration of interest}

None.

\section{Copyright and usage}

(C) The Royal College of Psychiatrists 2016. This is an open access article distributed under the terms of the Creative Commons Non-Commercial, No Derivatives (CC BY-NC-ND) licence.
South Africa has a 12 -month prevalence estimate of $16.5 \%$ for common mental disorders (anxiety, mood and substance use disorders), ${ }^{1}$ with almost a third $(30.3 \%)$ of the population having experienced a common mental disorder in their lifetime. ${ }^{2}$ These estimates are relatively high when compared with international prevalence estimates of the WHO (World Health Organization) World Mental Health surveys. ${ }^{3}$ As is the case internationally, ${ }^{4,5}$ the treatment gap in South Africa is also high, with only one in four people with a common mental disorder receiving treatment of any kind. For those with psychotic disorders, although identification and access to treatment is better, there are insufficient resources at community level for promotion of recovery. ${ }^{6,7}$ The aim of the formative phase of the PRogramme for Improving Mental health carE (PRIME) in South Africa was to (a) develop a mental healthcare plan (MHCP), customised to local conditions at district level that provides acceptable and feasible collaborative care packages for depression, alcohol use disorders and schizophrenia and can be integrated into existing service delivery platforms; (b) identify the human resource mix to deliver the MHCP and develop implementation tools to support and facilitate scale up of the packages; and (c) identify potential barriers to implementation at scale. The reason for the focus on these conditions is their relatively high burden of disease and disability and evidence of cost-effective interventions for their treatment. $^{8}$

\section{Method}

\section{Context}

Although South Africa is an upper-middle-income country, there are large disparities in wealth and access to resources. Within the health sector, these disparities are reflected in inequities between private and public health provision. Private healthcare, funded through private health insurance and out of pocket payments, serves approximately $16 \%$ of the population, compared with about $84 \%$ served by public healthcare. Yet gross domestic product spend on each is similar ( $4.1 \%$ and $4.2 \%$ respectively). ${ }^{9}$ To redress these inequities, South Africa is phasing in (over 14 years) a national health insurance system, to ensure universal access to appropriate, efficient and high-quality health services. ${ }^{9}$ The introduction of national health insurance involves an overhaul of services as well as systems to support service delivery. Notably, at the district-service level is re-engineering of primary healthcare. This includes the establishment of district specialist clinical teams to provide support to ward-based primary healthcare teams. The latter comprise primary healthcare staff at fixed primary healthcare facilities as well as community outreach teams consisting of a professional nurse and community health workers. ${ }^{10}$

Embedded within the national health insurance system, is the introduction of integrated chronic disease management (ICDM) to meet the needs of the rising number of patients with multiple chronic diseases associated with the roll-out of antiretroviral therapy and a burgeoning non-communicable diseases epidemic. ${ }^{11}$ At the facility level, the ICDM aims to strengthen the quality of care for chronic conditions through: (a) consolidating services for all chronic care patients, including those with communicable and non-communicable diseases, into a single delivery point; and (b) strengthening clinical decision support through the adoption of an integrated set of nurse-led clinical guidelines developed for the identification and management of multiple chronic diseases, called Primary Care 101 (PC101). ${ }^{12}$ At community level, community outreach teams support clinically stable patients in 
order to promote self-management. At a population level, health promotion and population screening are envisaged to promote an informed and activated population. ${ }^{13}$ Within this context, mental health is gaining ground as a public health priority. It is increasingly understood to be integral to the delivery of chronic care given that depression and alcohol misuse compromise prevention efforts as well as adherence to treatment. ${ }^{14-16}$

Legislative and policy developments specific to mental health include the introduction of a new Mental Health Care Act (No 17 of 2002) in 2004, as well as a new national mental health policy framework and strategic plan (2013-2020). Both promote decentralised and integrated care through task sharing. A noteworthy development that will enable implementation is the introduction of specialist district mental health teams expected to play a public mental health role.

Against this background, the South African national Department of Health advised that PRIME in South Africa focus on integrating mental health services for depression and alcohol use disorders into the ICDM service delivery platform given departmental priorities to reduce mortality as a result of chronic conditions, including HIV/AIDS. In light of service gaps in community-based psychosocial rehabilitation for patients with schizophrenia, ${ }^{6}$ this became a further focus of PRIME in South Africa. Ethical approval for the formative phase of PRIME, including the pilot study, was obtained from the University of KwaZulu-Natal Ethics Committee (HSS/0880/011 and BE 317/13) and the Human Research Ethics Committee of the Faculty of Health Sciences, University of Cape Town (REC Ref: 412/2011). All participants involved in semi-structured interviews consented to participating in the studies using approved informed consent procedures.

\section{Study site}

The Dr Kenneth Kaunda District (DKK) in the North West Province was chosen as the study site by the Department of Health as it is one of three districts where ICDM is being piloted in the country, and is a pilot site for national health insurance and the re-engineering of primary heathcare. DKK is in the southern part of the North West Province, which is located immediately west of the populous Gauteng province. Please see online Fig. DS1 for a map of South Africa with the location of DKK highlighted. DKK comprises four subdistricts, with a population of approximately 796823 , the majority of whom (90\%) are urban. The main economic activities are mining and agriculture. Public health facilities include regional hospitals, primary healthcare facilities and one specialist in-patient mental health facility (details are contained in online Table DS1). Private healthcare facilities are also available but were not the focus of study given the emphasis on integrating mental health into the public service ICDM service delivery platform.

\section{Research approach}

A mixed methods approach to the formative phase was used: a situational analysis; theory of change (ToC) workshops; qualitative interviews with service managers, service providers, patients and carers; and piloting of the preliminary MHCP in one clinic.

\section{Situational analysis}

A generic situational analysis tool developed for use across all PRIME country sites (downloadable from http://www.prime.uct. ac.za/images/prime/PRIME_Final_Situational_analysis_Tool.pdf) was adapted for the South African site. This tool required that information be gathered on a range of contextual issues such as burden of disease; mental health policies, plans and legislation; estimated treatment coverage for mental and neurological disorders; available resources and mental health information systems. Data were collected via secondary data sources at national and district level. The results of this situational analysis are reported elsewhere. ${ }^{17}$

\section{Theory of change workshops}

Participatory theory of change (ToC) workshops were held with key stakeholders including service managers, service providers and patients to develop a MHCP for the district. ToC provides a useful framework for guiding the development and ownership of complex health interventions such as an MHCP, starting with the intended impact and working backwards to identify the outcomes to achieve the impact, and the inputs and processes needed to achieve these outcomes. ${ }^{18}$ A more detailed account of the process is provided by Breuer et al. ${ }^{19}$

Discussion in the ToC workshops centred on how key outcomes identified in the cross-country $\mathrm{ToC}$ map ${ }^{20}$ could be achieved in the district for each priority condition. At the organisational level the key cross-country packages comprised engagement and mobilisation, programme implementation and management and capacity building. At the facility level, they comprised mental health literacy, identification and diagnosis, drug treatment, psychosocial interventions and continuing care. The community level included mental health literacy, detection and referral, adherence support, rehabilitation and mobilisation.

In total three $\mathrm{ToC}$ workshops were held over a period of 6 months from March to August 2012 to develop the initial plan. One workshop involved service providers and managers from facility level to the national level $(n=26)$; one was with community-based service providers and patients $(n=21)$; and one combined the above two groups $(n=31)$, which merged and refined the ToC maps into a final one, drawing on information from the formative qualitative interviews as well.

\section{Qualitative formative interviews}

Formative qualitative interviews were undertaken with key stakeholders to maximise the social and cultural fit of the emergent MHCP, including an acceptable human resource mix and associated tools to support implementation. All interviews were audiotaped. In total there were 79 individual interviews with patients $(n=70)$ and caregivers $(n=9) ; 47$ with service providers made up of 25 individuals and 4 focus groups $(n=22)$. All interviews were audiotaped. The interviews were translated where necessary with back translation checks applied and transcribed verbatim. The transcripts were analysed with the help of NVivo 10 qualitative data analysis software, using framework analysis by stakeholder group..$^{21}$ This involved a number of steps including: (a) reading and re-reading the transcripts; (b) the development of a coding framework based on the interview questions; (c) coding of the data, with emergent themes being added to the coding framework during this coding process; (d) summarising the responses from the respondents across each theme; and (e) interpreting the final themes in light of what they suggested for service planning and interventions. The results of the formative interviews are reported by stakeholder group in detail in a number of published articles. ${ }^{22-24}$

\section{Draft collaborative care packages}

The situational analysis, ToC workshops and formative interviews informed the development of preliminary collaborative care packages depicted in Fig. 1. At the community level, improved 
identification for all the disorders was to be achieved through the second phase of the Department of Health community health worker outreach team training programme, which includes training in identification and referral of people with mental disorders. These community health worker outreach teams are also responsible for tracing all patients with chronic care needs who default on treatment. At primary healthcare facility level, improved identification and diagnosis of comorbid depression, alcohol use disorders and schizophrenia by primary healthcare nurses and doctors was to be achieved through strengthening the mental health guidelines of PC101 to ensure alignment with the WHO's Mental Health Gap Action Programme (mhGAP) algorithms (subsequently referred to as PC101+); adding a psychoeducational page for the 'stressed/ miserable patient'; and adding additional mental health cases to the PC101 training.

For the depression package, patients with mild symptoms were to be provided with basic psychoeducation using the 'stressed/miserable patient' page; patients with moderate to severe depression were to be referred to a primary healthcare doctor for initiation of antidepressant medication and/or to a facility-based lay counsellor for initiation of individual or group counselling using the PRIME South Africa depression counsellor guidelines adapted from a local intervention using evidence-based approaches of interpersonal therapy and cognitive-behavioural techniques. ${ }^{25,26}$ (The term 'lay counsellor' is used in the South African context to differentiate this group from other counsellors who have formal qualifications in counselling. 'Lay counsellors' typically receive minimal training in counselling to provide a limited and prescribed service.) After 8 weeks of counselling (the optimum number used in an effective collaborative task sharing intervention for depression in India ${ }^{27}$ ) patients were to be reassessed by a primary healthcare nurse using PC101+ and referred onwards for specialist care if necessary. Patients on medication were expected to be monitored on a monthly basis when they come to collect their repeat medication.

For the alcohol misuse package, primary healthcare nurses/ doctors were expected to provide psychoeducation on what constitutes acceptable levels and patterns of alcohol use for patients with harmful or hazardous drinking using guidelines introduced into PC101+. This has been shown to be an effective strategy for reducing alcohol consumption globally. ${ }^{28}$ mhGAP guidelines for the management of alcohol withdrawal at primary healthcare level were included in $\mathrm{PC} 101+$, with onward referral to the local district hospital for detoxification and specialised rehabilitation services.

In the case of the schizophrenia package, according to the Mental Health Care Act, ${ }^{29}$ patients with acute conditions are admitted to the nearest designated district hospital that provides $72 \mathrm{~h}$ observation and stabilisation. If necessary they are then referred onwards to a specialist mental health facility for diagnosis and initiation of treatment. On being discharged, patients are expected to attend their nearest primary healthcare facility as a chronic care patient for ongoing medication. In the PRIME collaborative care package, these stabilised patients were to be referred to community-based psychosocial rehabilitation groups facilitated by auxiliary social workers from the Department of Social Development/local Mental Health Society (a Mental Health non-governmental organisation (NGO)). Recent evidence attests

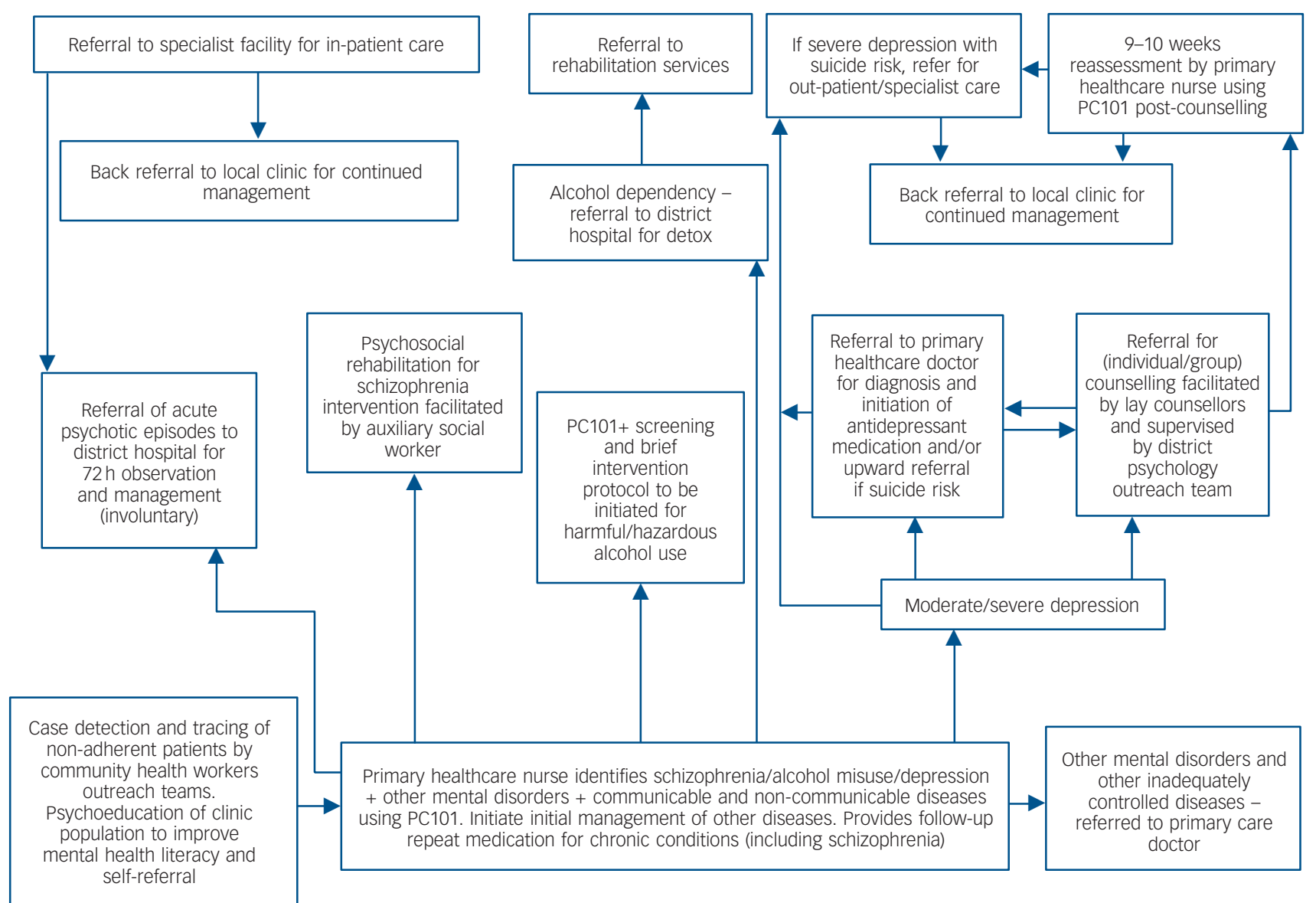

Fig. 1 Collaborative care packages for schizophrenia, alcohol misuse and depression. 
to the effectiveness of community-based task sharing approaches for reducing disability and symptoms of schizophrenia in India. ${ }^{30}$

\section{Pilot study}

These collaborative care packages were piloted from August to November 2013 in one clinic in DKK identified by the district management team. The identified clinic is situated in a peri-urban area of the district and saw an average of 3000 patients per month in 2013. Implementation involved a series of training workshops for personnel who formed part of the human resource mix required to deliver the packages of care.

Evaluation and monitoring of the implementation of the pilot involved monitoring implementation via implementation logs as well as process evaluation interviews. Process evaluation interviews were conducted at 3-month follow-up with service providers trained to deliver the intervention packages and patients who received the interventions. The purpose of the qualitative process evaluation interviews was to gain an understanding of individual's experience of delivering and receiving the interventions as well as bottlenecks that emerged and reasons for these. Participants included primary healthcare nurses $(n=4)$, lay counsellors $(n=4)$, auxiliary social workers $(n=2)$, patients who received counselling for depression $(n=6)$, patients who attended psychosocial rehabilitation groups $(n=6)$ and caregivers of patients attending psychosocial rehabilitation groups $(n=4)$. The interviews were translated and transcribed verbatim. Framework analysis, described previously, was used to analyse the data.

\section{Results}

For depression, over a 3-month period, only 15 patients were identified by the primary healthcare nurses and referred to the counsellors for depression. Two-thirds of patients referred $(n=10)$ presented for their counselling appointment and of these, 7 attended follow-up counselling sessions following the initial session. There were no recorded referrals to the primary healthcare doctor. No patients with alcohol use disorders were identified. For schizophrenia, 19 patients who had been down-referred from the psychiatric hospital to receive their follow-up medication from the clinic were identified from the clinic records. Of these, only nine were attending the clinic for their follow-up medication of which six eventually attended the psychosocial rehabilitation group on a regular basis, with one dropping out. Only one caregiver attended the caregiver sessions.

The bottlenecks identified by the pilot study thus included a paucity of referrals by primary healthcare nurses to the lay counsellors for depression counselling; minimal identification of alcohol use disorders; poor follow-up of counselling referrals made by counsellors; a high default rate of patients receiving follow-up medications for mental illness at the primary healthcare clinic, which limited the number of patients who could be accessed for psychosocial rehabilitation groups; and poor uptake of the psychosocial rehabilitation intervention by caregivers of patients with schizophrenia.

Reasons for these bottlenecks that emerged from the qualitative process interviews included the following.

(a) On the part of patients/caregivers, poor mental health literacy was a barrier to help-seeking for depression; defensiveness in divulging alcohol consumption was a barrier for identification of alcohol use disorders.

(b) On the part of nurses, barriers to identification and/or referral rates of depression and alcohol use disorders was a result of low self-confidence in ability to diagnose common mental disorders; unattended personal issues; focusing on underlying social problems and referral to social workers without attending to the presenting mental disorders; and lack of confidence in lay counsellor abilities.

(c) On the part of counsellors, in addition to unattended personal issues; marginalised status and unclear roles; low confidence; and poor suitability of some counsellors emerged as being reasons for poor follow-up of patients referred to them for counselling.

(d) Structural and organisational challenges that impeded identification and/or referral of depression or alcohol use disorders by nurses included high patient loads and space constraints that limited consultation.

(e) Space constraints also emerged as limiting confidentiality of counselling.

(f) With regard to the psychosocial rehabilitation group intervention, a high default rate and poor tracing of individuals who defaulted limited the number of patients who could be referred to the groups by the primary healthcare nurses.

A feedback meeting was held with key stakeholders including district managers, facility managers, service providers and patients in February 2014 to discuss the results of the pilot study, collectively identify strategies to overcome emergent bottlenecks and adapt the MHCP following the approach used by the International Health Institute for health systems strengthening. This approach uses a continuous quality improvement approach to bring healthcare facilities together at regular intervals to jointly identify and solve bottlenecks that emerge in the improvement of health system innovations. ${ }^{31}$

To aid roll-out, the implementation tools have been strengthened to include an implementation toolkit comprising implementation guidelines for district and facility managers, providing a step-by-step guide for the implementation and integration of mental health services at facility level. This toolkit also incorporates change management to orientate managers and service providers to the ethos and organisational needs of the collaborative chronic care, including providing more containing leadership and stress management; establishing targets for identification and treatment of priority mental disorders; and role clarification of the different team members where the primary healthcare nurse is designated as the case manager; lay counsellors designated to provide counselling for depression; auxiliary social workers are designated to provide psychosocial rehabilitation for patients with schizophrenia; and community health workers' role in following up patients who have defaulted on treatment and/or psychosocial intervention is emphasised. The need to strengthen the existing employee wellness programme at an organisational level was also highlighted.

The piloting process also informed how the guidelines and training materials in the toolbox needed to be strengthened. Noteworthy is modification of the alcohol use disorders guidelines to take into account patients' defensiveness in acknowledging high levels of alcohol use as patients with chronic care needs are often told to abstain from drinking alcohol; the introduction of waiting room educational talks in addition to information leaflets to improve mental health literacy; and the strengthening of referral documentation and information collected on the priority conditions. All the training materials, guidelines and resources comprise an 'implementation toolbox' for integrating mental healthcare at district level in South Africa (see online Table DS2 for a comprehensive list). A summary of the findings of the piloting process and how they informed modifications to the 
MHCP and adaptation of the implementation tools is provided in Table 1.

The final MHCP is depicted in Tables 2-4. It comprises core intervention packages at the organisational (Table 2), facility (Table 3) and community (Table 4) levels identified through the ToC process to achieve the identified outcomes along the continuum of care (see Method), with the addition of employee wellness at the organisational level. These packages comprise the human resources required to deliver each package as well as resources/mechanisms to aid implementation.

Based on the collaborative care models for the different disorders, the implications for the human resource mix, their roles and responsibilities and associated resources/mechanisms required to implement the plan at scale are depicted in Table 5. Identification of the human resource mix is important to inform core competencies and curricula of training programmes; and the role of regulatory and accreditation bodies in ensuring the production of a workforce equipped with the necessary competencies for scale up.

\section{Discussion}

The advantages of integrating mental health into existing service delivery platforms include the opportunity for the provision of holistic care; reduction in stigma; and leverage of existing resources to promote efficiency and greater effectiveness of health interventions. ${ }^{32}$ Given synergies with chronic care, unlike other country plans in this supplement, South Africa is in the fortunate position of being able to scaffold off the introduction of ICDM, with chronic care embracing a collaborative patient-centred approach, central to integrated mental healthcare. We have structured our discussion using the framework suggested by Patel et al on how to integrate mental health into other healthcare platforms. $^{32}$ This framework incorporates three aspects:

Table 1 Bottlenecks identified in pilot study, reasons and modifications to the mental healthcare plan (MHCP) and implementation tools

Bottleneck and reasons $\quad$ Modifications to MHCP and implementation tools

Paucity of referrals for depression to lay counsellors by primary healthcare nurses and minimal identification of alcohol use disorders

Organisational

High patient loads

Limited time and space for consultation needed to identify symptoms Weak information system for mental health

Primary healthcare providers

Biomedical orientation

Unattended personal issues

Psychiatric stigma

Perception of depression/alcohol use disorders as a social problem requiring referral to a social worker

Patients

Low mental health literacy

Defensiveness in divulging alcohol consumption

Low follow-up of counselling referrals by counsellors

Organisational

Unclear role clarification of lay counsellor roles

Marginalised status

Primary healthcare providers

Low self-esteem

Change management for district/facility managers and service providers Inclusion of depression on chronic care form

Facility target for identification of depression/alcohol use disorders

Alcohol use disorders PC101+ guidelines strengthened

Strengthened employee assistance programme

Lay counsellors

Unattended personal issues

Poor suitability to counselling role

Patients

Low mental health literacy

Waiting room educational talks

Information leaflets

Inclusion of primary healthcare nurse as case managers

Role clarification of primary healthcare nurses and lay counsellors in PC101+ training

Role clarification and stress management in lay counsellor training Selection of dedicated lay counsellors

Waiting room educational talks

Information leaflets

High rate of defaulting on follow-up medication

Organisational

ICDM (no dedicated queue or nurse for psychiatric patients)

Poor tracking of defaulters

Primary healthcare providers

Psychiatric stigma

Poor understanding of severe mental illness

Patients

Low mental health literacy

Strengthened role of community health workers in tracking patients with mental disorders

Strengthened orientation to mental health in PC101+

Psychosocial rehabilitation intervention Information leaflets

Low uptake of psychosocial rehabilitation intervention by caregivers

Organisational

Poor community outreach to families

Primary healthcare providers

Poor understanding of the need to provide psychoeducation to families

Caregivers

Low mental health literacy

Psychiatric stigma

Strengthened role of community outreach to provide psychoeducation

Strengthened PC101+ training

Focused engagement of caregivers by community outreach teams prior to programme

PC101, Primary Care 101; ICDM, integrated chronic disease management. 

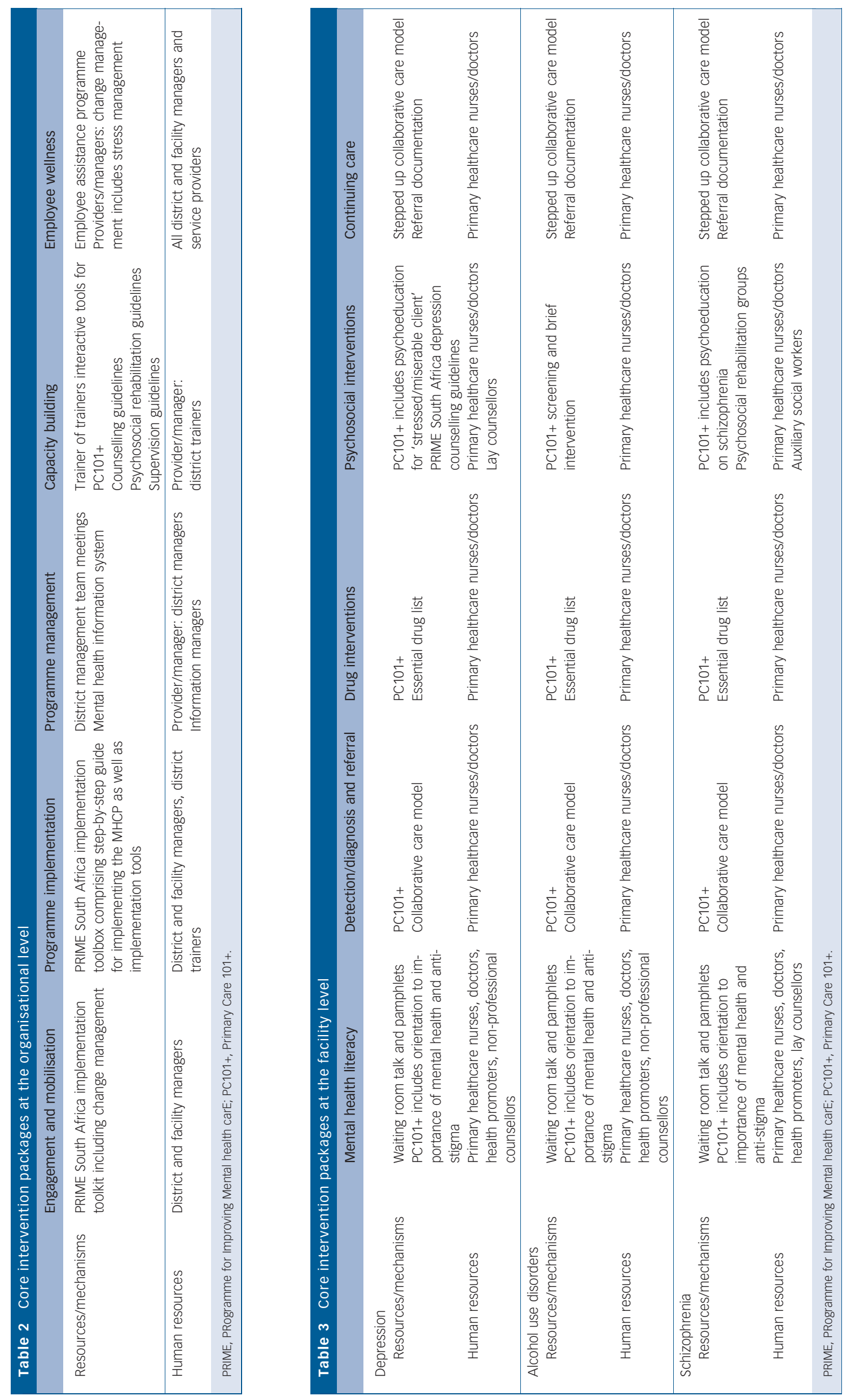
assessment and customisation; tasks and human resources; and standardisation.

First, assessment and customisation includes active collaboration of service managers and providers to accurately assess what can feasibly be delivered by the platform, and what lies beyond the scope of the platform and needs to be referred. The participatory nature of the $\mathrm{ToC}$ and quality improvement workshops helped facilitate customisation of the MHCP but a number of issues that emerged from the pilot were overlooked.

These include first, that the collaborative care models adopted by the MHCP require a paradigm shift in the approach to care. ${ }^{33}$ Although ICDM provides a potentially enabling platform for integration of mental health, staff need to be orientated to this approach. Change management workshops to orientate facility managers and service providers to the ICDM and integrated mental health have thus been included in the revised MHCP at an organisational level.

Second, with respect to the increased burden of emotional labour that accompanies mental healthcare, to minimise burnout and assist service providers who may experience mental health difficulties of their own, the revised MHCP includes (a) strengthening the employee assistance programme: and (b) instilling more containing leadership including stress management (included in the change management workshops).

Third, in relation to role clarification, the human resource mix and associated skills sets and resources to achieve the plan are contained in Table 5. Notwithstanding evidence of the effective use of lay health workers in other low- and middle-income countries to increase access to psychosocial interventions, ${ }^{34}$ poor role clarification and marginalised status of existing lay counsellors in the South African healthcare system ${ }^{35}$ resulted in two major difficulties: a lack of confidence and reticence to take on additional counselling duties on their part; and low referrals by primary healthcare nurses who did not trust their competencies to counsel patients effectively. This has been addressed in the implementation toolkit of the revised MHCP through: (a) more clearly identifying a case manager (primary healthcare nurse) responsible for monitoring patient progress at primary healthcare level (which has been a need identified in the MHCP in India ${ }^{36}$ in this supplement); and (b) providing greater role clarification of lay counsellors.

A further difficulty has been harnessing human resources from other sectors, particularly auxiliary social workers from the Department of Social Development and NGO sector to facilitate psychosocial rehabilitation groups. Greater formal collaboration between the Department of Health and Department of Social Development at national and provincial level has been identified as a strategy to address this.

Standardisation refers to monitoring of patient progress to assess whether care needs to be adjusted or 'stepped up. ${ }^{32}$ This approach is commonly used in chronic care to monitor remission and obviate patients falling through the cracks. It has been incorporated into the collaborative care models for all three conditions but was not evaluated in the pilot study as no patient reached the point of needing upward referral.

In relation to the human resource requirements and costing of the PRIME South Africa MHCP, readers are referred to the crosscountry costing paper in this supplement, ${ }^{37}$ where the number of additional full-time equivalent staff and associated costs for increasing coverage for the priority conditions in the MHCP are estimated. Given that mental health is part of ICDM in South Africa, the cost of increasing coverage of integrated mental healthcare in South Africa ${ }^{37}$ will have to be borne by the existing primary healthcare budget and thus have to compete with other chronic care priorities. For planners to see the value of integrated mental 
Table 5 Human resource mix for PRogramme for Improving Mental health carE (PRIME) South Africa mental healthcare plan

Human resource $\quad$ Function/Services Resource/mechanism/tool

District/subdistrict management tier

District management team (chief director; Management of programmes for example director: primary healthcare services; director: hospital services; manager: subdistrict director: primary healthcare services; district manager: training) Subdistrict team (directors: primary healthcare services; community health services; coordinators: chronic care/ mental health; counselling) Information officer maternal health, HAST (HIV/AIDS/sexually transmitted infections/tuberculosis)

Ensure collaboration with other services Ensure capacity building

Management of all programmes including chronic care

District management team meetings

Training programmes

Plan, manage and monitor the mental health components of the district information system

Subdistrict management team meetings

PRIME implementation toolkit including change management workshop

Social cluster meetings to promote intersectoral collaboration Existing MHIS

Provincial indicator data-set, district health information system

Patient register in terms of Section 39 of the Regulations to the Mental Health Care Act No 17 of 2002 for health establishments that render mental health services

District/subdistrict pharmacist

Ensuring sufficient stock of psychotropic medication

Dispensing of medication and distribution

to primary healthcare Packaging of psychotropic medication for patients

\section{Tertiary hospital tier Psychiatrist}

Clinical psychologist

Psychiatric nurse

Clinical social worker

Occupational therapist Pharmacist

Dietician

Neurologist (part time)

Information officer

District hospital tier

Clinical psychologist

Medical officers

Psychiatrist (part time)
Assessment, diagnosis and holistic treatment Hospital protocol

(including psychiatric treatment) of in-patients/ Standard treatment guidelines and essential drug list out-patients hospital level adults 2006

Consultation-liaison service for primary health-

care doctors and other mental health specialists

(outreach and support)

Training and supervision of registrars

Assessment, diagnosis and psychological treatments for in-patients and out-patients

Psychoeducation of patients/families

Training of intern psychologists

In-patient care/out-patient nursing care

Psychosocial rehabilitation

Psychoeducation of families

Family assessment and psychoeducation

Psychoeducation of families

Placement in alternative accommodation

and/or sheltered workshops on discharge

Assist psychiatric patients with applications

for disability grants

Assessment and psychosocial rehabilitation

Orders and dispenses medication

Prepares menus

Assesses electroencephalograms

Capture and manage patient records

Assessment, diagnosis and psychological treatments for in-patients (emergency admissions, $72 \mathrm{~h}$ observation of involuntary patients, short-term acute in-patient care and upward referral to tertiary specialist services

Psychological referral service for complicated cases and more severe mental illness Training of intern psychologists

Referral support to primary healthcare level Assessment, diagnosis and medical treatments Mental Health Care Act for mental disorders and other comorbid Hospital protocol

medical conditions for in-patients/out-patients Standard treatment guidelines and essential drug list for Detoxification for individuals with substance misuse

Assessment, diagnosis and psychiatric treatment for in-patients (emergency admissions, $72 \mathrm{~h}$ observation of involuntary patients, short-term acute in-patient care and upward hospital level adults 2006

Mental Health Care Act

Hospital protocol

Standard treatment guidelines and essential drug list for hospital level adults 2006

referral to tertiary spec services

Psychiatric referral service for confirmation and adjustment of diagnoses and treatment regimes for more complex psychiatric cases 
Table 5 Human resource mix for PRogramme for Improving Mental health carE (PRIME) South Africa mental healthcare plan (continued)

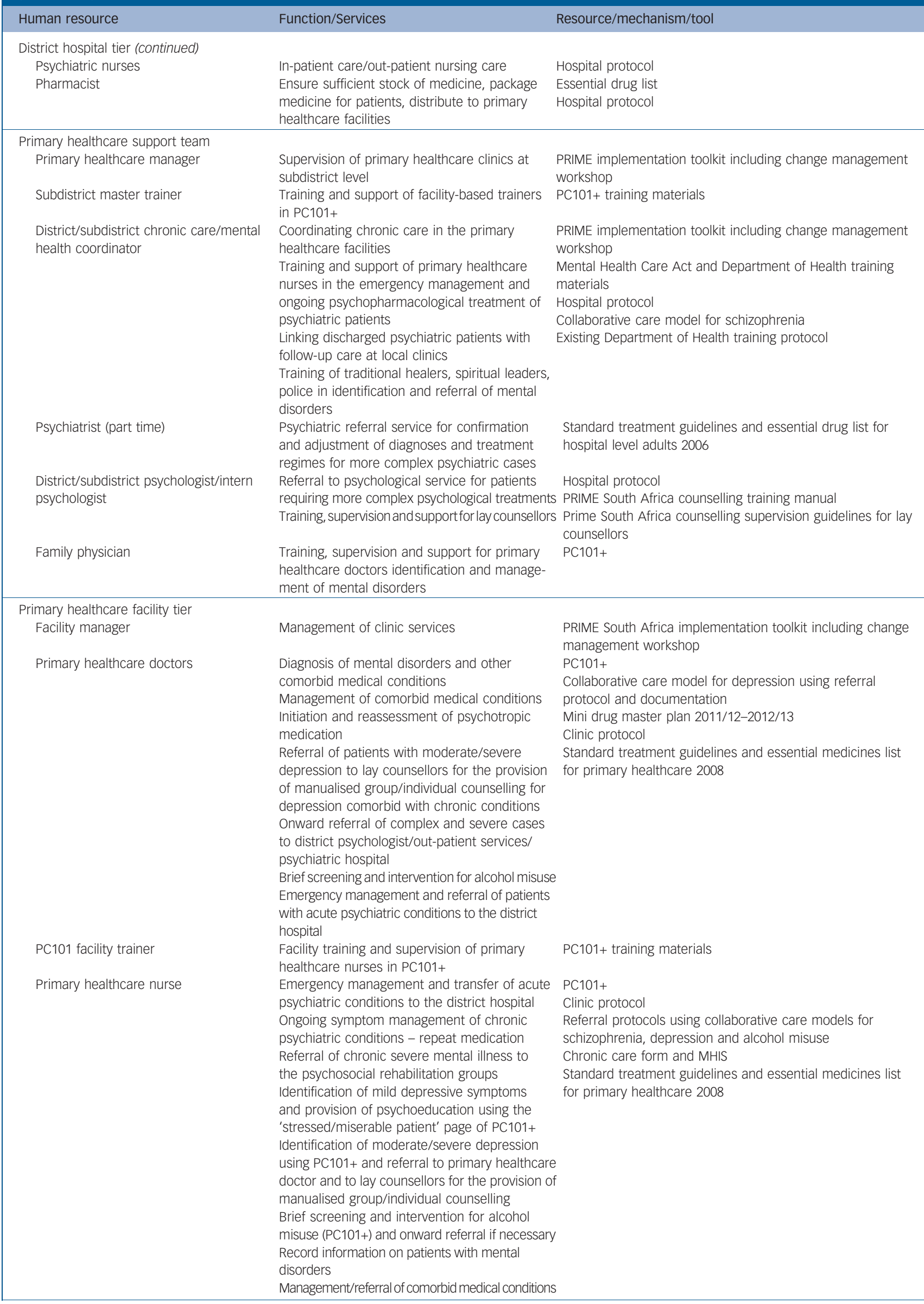




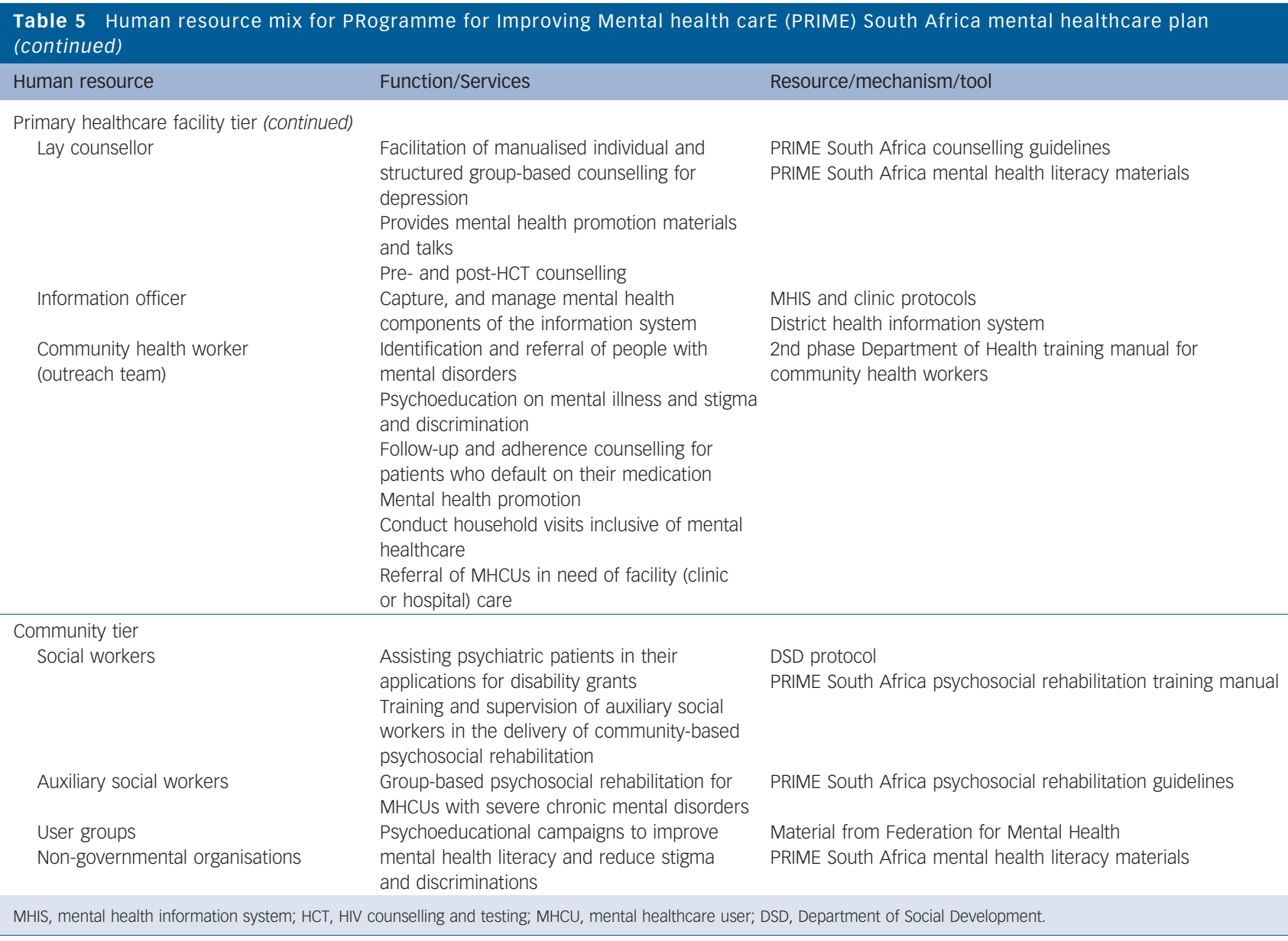

healthcare, the need for cost-benefit studies to show the cost savings and impact of integrated mental health on improved health outcomes in chronic care in South Africa emerged as a priority.

\section{Limitations}

An obvious limitation is the lack of integration into a maternal and child health service delivery platform. This is of concern given the high rate of maternal depression in South Africa ${ }^{38}$ and the negative impact on child developmental outcomes. ${ }^{39}$ Reasons for this include the current focus of the Department of Health on the ICDM and that in South Africa, maternal and child health occurs through a different service delivery platform in primary healthcare and would require the development of a different MHCP. This remains a challenge for the future. A further limitation is the difficulty in reaching men with alcohol misuse. Alcohol use is more prevalent in men in South Africa ${ }^{40}$ and given they comprise a minority (approximately a third) of primary healthcare clinic attendees in the North West province, the need to engage other health service delivery platforms such as private healthcare provided on the mines is indicated. An additional limitation of the MHCP is that there was minimal engagement with traditional healers in the development of the plan. Given that a large number of people with mental disorders consult traditional healers in South Africa, engaging traditional healers in the collaborative care models is an important task as we move forward.

\section{Recommendations}

The recent adoption of a national mental health policy framework that embraces decentralised care and task sharing, together with mental health gaining ground as a public health priority, bodes well for future scaling up of the integrated mental healthcare services in South Africa. Although the PRIME South Africa MHCP does not serve as a blueprint for scale up to other districts given the great variety in resources available across districts in South Africa, the approach and implementation tools developed should aid this process.

An important consideration in scale up is the additional time demands on an already burdened system, reflected as an increase in the number of full-time equivalent's required for scaling up integrated care in the costing of the plan. ${ }^{37} \mathrm{~A}$ recommendation would be to use the introduction of national health insurance in South Africa to leverage additional resources for mental healthcare. To strengthen this possibility, cost-benefit studies demonstrating the health benefits and cost savings of integrated mental health are needed. This is especially important, given the lack of a dedicated budget for mental health within ICDM and the need to compete with other priority conditions for resources.

Inge Petersen, PhD, School of Applied Human Sciences, University of KwaZulu-Natal, Durban, South Africa; Lara Fairall, PhD, Knowledge Translation Unit, University of Cape Town, Cape Town, South Africa; Arvin Bhana, PhD, Tasneem Kathree, MSocSci, One Selohilwe, MSOcSci, Carrie Brooke-Sumner, MSC, School of Applied Human Sciences, University of KwaZulu-Natal, Durban, South Africa; Gill Faris, MPhil, Human Sciences, University of KwaZulu-Natal, Durban, South Africa; Gill Faris, MPhil,
Knowledge Translation Unit, University of Cape Town, Cape Town, South Africa; Erica Breuer, MPH, Alan J Flisher Centre for Public Mental Health, Department of Psychiatry and Mental Health, University of Cape Town, Cape Town, South Africa; Nomvula Sibanyoni, MSocSci, National Department of Health, Pretoria, South Africa; Crick Lund, PhD, Alan J Flisher Centre for Public Mental Health, Department of Psychiatry and Mental Health, University of Cape Town, Cape Town, South Africa, and Centre for Global Mental Health, Institute of Psychiatry, Psychology and Neuroscience, King's College London, UK; Vikram Patel, PhD, Centre for Global Mental Health, London School of Hygiene and Tropical Medicine, London, UK and Centre for Mental Health, the Public Health Foundation of India, India

Correspondence: Inge Petersen, School of Applied Human Sciences, School of Applied Human Sciences, Howard College, University of KwaZulu-Natal, Durban, 4000, South Africa. Email: peterseni@ukzn.ac.za

First received 1 Jul 2014, final revision 19 Jan 2015, accepted 21 Jan 2015 


\section{Funding}

This study is an output of the PRogramme for Improving Mental health carE (PRIME). The material has been funded by UK aid from the UK government. However, the views expressed do not necessarily reflect the UK government's official policies.

\section{Acknowledgements}

We would like to thank all the participants from the district who gave freely of their time to participate in the development and piloting of the mental healthcare plan.

\section{References}

1 Williams DR, Herman A, Stein DJ, Heeringa SG, Jackson PB, Moomal H, et al. Twelve-month mental disorders in South Africa: prevalence, service use and demographic correlates in the population-based South African Stress and Health Study. Psychol Med 2008; 38: 211-20.

2 Stein DJ, Seedat S, Herman A, Moomal H, Heeringa SG, Kessler RC, et al. Lifetime prevalence of psychiatric disorders in South Africa. Br J Psychiatry 2008; 192: 112-7.

3 Kessler RC, Aguilar-Gaxiola S, Alonso J, Chatterji S, Lee S, Ormel J, et al. The global burden of mental disorders: an update from the WHO World Mental Health (WMH) surveys. Epidemiol Psichiatr Soc 2009; 18: 23-33.

4 World Health Organization. Global Burden of Mental Disorders and the Need for a Comprehensive, Coordinated Response from Health and Social Sectors at the Country Level. WHO, 2011 (http://apps.who.int/gb/ebwha/pdf_files/ EB130/B130_9-en.pdf).

5 Kohn R, Saxena S, Levav I, Saraceno B. The treatment gap in mental health care. Bull World Health Organ 2004; 82: 858-66.

6 Petersen I, Lund C. Mental health service delivery in South Africa from 2000 to 2010: one step forward, one step back. S Afr Med J 2011; 101: 751-7.

7 Lund C, Kleintjes S, Kakuma R. Public sector mental health systems in South Africa: inter-provincial comparisons and policy implications. Soc Psychiatry Psychiatr Epidemiol 2010; 45: 393-404.

8 Lund C, Tomlinson M, Da Silva M, Fekadu A, Shidhaye R, Jordans M, et al. PRIME: a programme to reduce the treatment gap for mental disorders in five low and middle-income countries. PLOS Med 2012; 9: e1001359.

9 Department of Health. National Health Insurance in South Arica. Policy Paper Department of Health, 2011.

10 Matsotso MP, Fryatt R. National Health Insurance: the first 18 months. In South African Health Review 2012/13 (eds A Padarath, R English): 21-33. Health Systems Trust, 2013.

11 Mayosi BM, Flisher AJ, Lalloo UG, Sitas F, Tollman SM, Bradshaw D. The burden of non-communicable diseases in South Africa. Lancet 2009; 374: 934-47.

12 Department of Health. Primary Care 101. Department of Health, 2012.

13 Asmall S, Mahomed $\mathrm{OH}$. The Integrated Chronic Disease Management Manual. Department of Health, 2013.

14 Gonzalez JS, Batchelder AW, Psaros C Safren SA. Depression and HIV/AIDS treatment nonadherence: a review and meta-analysis. J Acquir Immune Defic Syndr 2011; 58: 181-7

15 Moussavi S, Chatterji S, Verdes E, Tandon A, Patel V, Ustun B. Depression, chronic diseases, and decrements in health: results from the World Health Surveys. Lancet 2007; 370: 851-8.

16 Neuman MG, Schneider M, Nanau RM, Parry C. Alcohol consumption, progression of disease and other comorbidities, and responses to antiretroviral medication in people living with HIV. AIDS Res Treatment 2012 2012: 751827

17 Hanlon C, Luitel NP, Kathree T, Murhar V, Shrivasta S, Medhin G, et al. Challenges and opportunities for implementing integrated mental health care: a district level situation analysis from five low- and middle-income countries. PloS One 2014; 9: e88437.

18 Vogel I. Review of the Use of 'Theory of Change' in International Development. UK Department of International Development, 2012.

19 Breuer E, De Silva M, Fekadu A, Luitel NP, Murhar V, Nakku J, et al. Using workshops to develop Theories of Change in five low and middle income countries: lessons from the Programme for Improving Mental Health Care (PRIME). Int J Ment Health Systems 2014; 8: 15.
20 Breuer E, De Silva MJ, Shidaye R, Petersen I, Nakku J, Jordans MJD, et al. Planning and evaluating mental health services in low- and middle-income countries using theory of change. Br J Psychiatry 2015, in press (doi: 10.1192/bjp.bp.114.153841).

21 Ritchie R, Spencer L. Qualitative data analysis for applied policy research. In Analyzing Qualitative Data (eds A Bryman, RG Burgess): 173-94. Routledge, 1994

22 Petersen I, Hanass Hancock J, Bhana A, Govender K, Members of Programme for Improving Mental Health Care. Closing the treatment gap for depression co-morbid with HIV in South Africa: voices of afflicted women. Health 2013; 5: $557-66$.

23 Kathree T, Selohilwe OS, Bhana A, Petersen I. Perceptions of postnatal depression and health care needs in a South African sample: the "mental" in maternal health care. BMC Women's Health 2014; 14: 140.

24 Brooke-Sumner C, Petersen I, Lund C. Perceptions of disability and recovery among mental health services users and their caregivers in the North West Province, South Africa: lessons for development of a psychosocial rehabilitation programme. Afr J Disability 2014; 3: 1-10.

25 Petersen I, Hanass Hancock J, Bhana A, Govender K. A group-based counselling intervention for depression comorbid with HIV/AIDS using a task shifting approach in South Africa: a randomized controlled pilot study. J Affect Disord 2014; 158: 78-84.

26 Dua T, Barbui C, Clark N, Fleischmann A, Poznyak V, van Ommeren M, et al. Evidence-based guidelines for mental, neurological, and substance use disorders in low- and middle-income countries: summary of WHO recommendations. PLOS Med 2011; 8: e1001122.

27 Patel V, Weiss HA, Chowdhary N, Naik S, Pednekar S, Chatterjee S, et al. Effectiveness of an intervention led by lay health counsellors for depressive and anxiety disorders in primary care in Goa, India (MANAS): a cluster randomised controlled trial. Lancet 2010; 376: 2086-95.

28 Rehm J, Chisholm D, Room R, Lopez AD. Alcohol. In Disease Control Priorities in Developing Countries (2nd edn) (eds D Jamison, J Breman, A Measham, G Alleyne, M Claeson, DB Evans, et al.): 887-906. Oxford University Press, 2006.

29 Department of Health. Mental Health Care Act (2002). Department of Health, 2004

30 Chatterjee S, Naik S, John S, Dabholkar H, Balaji M, Koschorke M, et al. Effectiveness of a community-based intervention for people with schizophrenia and their caregivers in India (COPSI): a randomised controlled trial. Lancet 2014; 384: 1385-94.

31 Barker PM, McCannon CJ, Mehta N, Green C, Youngleson MS, Yarrow J, et al. Strategies for the scale-up of antiretroviral therapy in South Africa through health system optimization. J Infect Dis 2007; 196 (suppl 3): S457-63.

32 Patel V, Belkin GS, Chockalingam A, Cooper J, Saxena S, Unützer J. Grand challenges: integrating mental health services into priority health care platforms. PLOS Med 2013; 10: e1001448.

33 Petersen I. Comprehensive integrated primary mental health care for South Africa. Pipedream or possibility? Soc Sci Med 2000; 51: 321-34.

34 Thornicroft G, Tansella M. Are community mental health services relevant in low- and middle-income countries? Epidemiol Psychiatr Sci 2014; 23: 115-8.

35 Petersen I, Fairall L, Egbe CO, Bhana A. Optimizing lay counsellor services for chronic care in South Africa: a qualitative systematic review. Patient Educ Couns 2014; 95: 201-10.

36 Shidhaye R, Shrivastava S, Murhar V, Samudre S, Ahuja S, Ramaswamy R, et al. Development and piloting of a plan for integrating mental health in primary care in Sehore district, Madhya Pradesh, India. Br J Psychiatry 2015, in press (doi: 10.1192/bjp.bp.114.153700).

37 Chisholm D, Burman-Roy S, Fekadu A, Kathree T, Kizza D, Luitel NP, et al. Estimating the cost of implementing district mental healthcare plans in five low- and middle-income countries: the PRIME study. Br J Psychiatry 2015, in press (doi: 10.1192/bjp.bp.114.153866).

38 Hartley M, Tomlinson M, Greco E, Comulada WS, Stewart J, Roux I, et al. Depressed mood in pregnancy: prevalence and correlates in two Cape Town peri-urban settlements. Reprod Health 2011; 8: 9.

39 Walker SP, Wachs TD, Gardner JM, Lozoff B, Wasserman GA, Pollitt E, et al. Child development: risk factors for adverse outcomes in developing countries. Lancet 2007; 369: 145-57.

40 Peltzer K, Ramlagan S. Alcohol use trends in South Africa. J Soc Sci 2009; 18: $1-12$. 